\title{
Procedimiento para elaborar el plan de negocio en el área de alojamiento en pequeños hoteles
}

DOI: 10.22403/UQROOMX/TYP10/06

\author{
Berta Leidy González Valdés \\ Carlos Cristóbal Martínez Martínez \\ Facultad de Ingeniería Industrial y Turismo \\ Universidad Central "Marta Abreu” de Las Villas
}

\section{Inthe}

\section{RESUMEN}

Las tendencias actuales de las actividades del turismo, los viajes y la hospitalidad exigen un reto superior para los establecimientos de alojamiento turístico, los cuales se ven en la necesidad de buscar herramientas, métodos y procedimientos de gestión que les permitan adaptarse a las nuevas características del sector hotelero en el nivel mundial. En este trabajo se propone un procedimiento para la elaboración del plan de negocio en el área de alojamiento en pequeños hoteles, que pretende, fundamentalmente, vincular las tendencias actuales en cuanto a los elementos que deben formar parte del procedimiento, las necesidades de las entidades hoteleras y las particularidades de la gestión de alojamiento, integrando para ello los aspectos positivos de los autores consultados y excluyendo las insuficiencias detectadas.

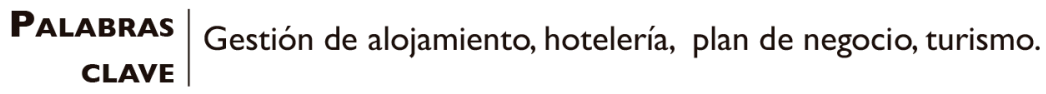

Recibido el 18 de enero de 2011

Aprobado el II de marzo de 20 I I

Correos electrónicos: bertagv@uclv.edu.cu•cristobalc@uclv.edu.cu 


\section{Introducción}

La industria turística en las últimas décadas ha contribuido en forma significativa al desarrollo económico, social y cultural de muchos países, y se ha convertido en un negocio altamente lucrativo para la mayoría de las entidades turísticas involucradas en la prestación de servicios (Figuerola, 1990; Álvarez, 200 I; Muñoz, 2004; Matos, 2005). En los últimos años se han intensificado los esfuerzos tendentes al logro de una gestión cada vez más eficiente de la empresa hotelera, con el propósito de una más adecuada orientación a la actividad turística y un óptimo aprovechamiento de los beneficios que brinda su desarrollo, que cada día se hacen más evidentes. Los establecimientos de alojamiento hotelero se presentan como el instrumento del sistema turístico capaz de satisfacer los requerimientos necesarios para la realización de los viajes (Matos, 2005), y, en concordancia con otros autores, se considera que hoy estas entidades presentan una disyuntiva de cambios y transformaciones, por lo que es necesario reflexionar hacia dónde se dirige y cuáles son los retos de la hostelería ante el nuevo milenio (Herrera, 2000; Martínez Martínez, 2003).

En este sentido, es preciso establecer un enfoque o mecanismo de gestión que asegure, de manera consciente y estable, altos niveles de calidad en la gestión de alojamiento en los establecimientos hoteleros en correspondencia con los valores y principios que conforman la cultura organizacional. La celeridad de los cambios y la falta de respuesta de muchos establecimientos de alojamiento turístico para adaptarse a estas transformaciones han provocado que se requieran cada vez más nuevas herramientas gerenciales (González Valdés y Martínez Martínez, 2009).Aspectos como entender mejor su servicio o producto, determinar sus objetivos, anticipar posibles problemas y fallas, clarificar de dónde vendrán sus recursos financieros, tener parámetros para medir el crecimiento de la entidad de alojamiento y la existencia de estrategias técnicamente fundamentadas exigen una gran agilidad y flexibilidad a la hora de tomar decisiones (Gallegos, 2002; Muñoz, 2004; Matos, 2005).

Entonces, surge la necesidad conceptual, metodológica y gerencial de introducir un instrumento que permita concretar las estrategias en términos técnicos, económicos, tecnológicos y financieros. Por ello, la elaboración del plan de negocio puede convertirse en una poderosa herramienta gerencial al permitir, a la vez que se diseña, observar riesgos, oportunidades y estimaciones diferentes, así como percibir las necesidades financieras y vincularlas con 
los parámetros proyectados del área de alojamiento del hotel. Lynn (2000), Martínez Martínez (2007), Martínez Martínez,Valdivia y Cruz (2007) y González Valdés (2008) identifican algunas razones por las que se decide realizar un plan de negocio:

- Asegurar que un negocio tenga sentido financiero y operativo antes de su puesta en marcha

- Buscar la forma más eficiente de llevar a cabo un proyecto

- Crear un marco que permita identificar y evitar posibles problemas antes de que ocurran, con el consiguiente ahorro de tiempo y recursos

- Prever necesidades de recursos y su asignación en el tiempo

- Evaluar el desempeño de un negocio en marcha

Se coincide con los criterios ofrecidos por otros autores en que, en el área de alojamiento de pequeños hoteles, donde las decisiones suelen estar concentradas en pocas personas, la elaboración de un plan de negocio tiene como ventaja adicional establecer una relación entre el humor cambiante (o los caprichos) de los gerentes y las decisiones de negocio tomadas con base en información y análisis (Wald, 2005; Terragno y Lecuona, 2006). El plan de negocio, como una herramienta para la gestión de alojamiento, está dirigido a constituir un medio fundamental para ser utilizado en entidades de nueva creación o en desarrollo, por lo que es importante su correcta elaboración a partir de procedimientos adecuados a las características de la organización (Lynn, 2000). Su empleo como herramienta de gestión para la actividad del área de alojamiento turístico contribuye a organizar la información y establecer por escrito las estrategias, políticas, objetivos y acciones que el área necesita como parte de su proyección, provocando insuficiencias en la gestión de alojamiento turístico.

\section{Requisitos para elaborar un plan de negocio en el área de alojamiento de pequeños hoteles}

A partir de los criterios de los diversos autores consultados, se propone que el plan de negocio constituye un informe cuyo objetivo principal será constatar por escrito la viabilidad económica y financiera y reflejar los objetivos a alcanzar, así como el potencial del área de alojamiento dentro del establecimiento hotelero (Díaz, 1994; Infante y Carballosa, 2004; Dezerega, 2004; Cruz, 2007). 
El plan de negocio se convierte entonces en una herramienta gerencial útil por cuanto hace posible organizar y gestionar eficientemente el alojamiento, empleando un plan que permite innovar en servicios y productos, con la seguridad de invertir de manera adecuada. En consecuencia con las fuentes consultadas, los autores de este trabajo proponen un conjunto de requisitos que debe tenerse en cuenta para confeccionar el plan de negocio en el área de alojamiento:

- Rigurosidad en la elaboración. Preparar un documento bien desarrollado, con datos exactos, proyecciones objetivas, y ajustado a los pasos establecidos.

- Brindar información pertinente y actualizada. Los datos a proporcionar deben ser precisos, fiables, que permitan el análisis adecuado, sea tanto de estadísticas o de cualquier investigación realizada, como de datos que manejan empíricamente los directivos.

- Formación/superación. Es importante recalcar con base en resultados reales la necesidad de que el nivel de preparación de todos los implicados en el plan de negocio se eleve en beneficio de la gestión de alojamiento del hotel.

- Buscar el desarrollo del mercado.Tratar de sacarle el máximo provecho al mercado no significa abusar de él sino saber aprovechar las oportunidades al satisfacer las necesidades de sus clientes reales y potenciales.

- Analizar el mercado. Hay que conocer en detalle a los clientes, sus características, gustos y preferencias, así como con qué frecuencia visitan el lugar, qué buscan y qué tan satisfechos están con los servicios recibidos, por qué lo eligen y quiénes son los competidores.

- Detectar sus puntos débiles y fuertes.Analizar la gestión de alojamiento de manera objetiva. Qué se hace bien y qué se hace mal, por qué y cómo solucionar estas deficiencias para manejar al máximo las fortalezas.

- Fijar un cronograma. La elaboración del plan de negocio no puede significar una serie de tareas improvisadas. Si se pretende utilizar como herramienta para mejorar la gestión, se le debe dar toda la importancia y organización que amerita el esfuerzo. Es necesario fijar fechas, actividades programadas, responsables y cumplimiento en cada etapa y paso propuestos como parte del procedimiento. 
El plan de negocio puede convertirse en un instrumento gerencial útil para el área de alojamiento, como lo demuestra su creciente utilización, comprobada mediante estudios efectuados por varios autores (Díaz, 1994; Lynn, 2000; Dezerega, 2004; Hernández y Zaragoza, 2004; Infante y Carballosa, 2004; Fontanez, 2005; Cruz, 2007; Torres Márquez, 2007; Martínez Martínez, 2007), aplicando técnicas y buenas prácticas obtenidas de las mejores experiencias aceptadas, nacionales e internacionales.

\section{Procedimiento propuesto para elaborar el plan de negocio en el área de alojamiento de pequeños hoteles}

Dado que se coincide con la mayoría de los autores en que el plan de negocio es una herramienta gerencial de gran utilidad, es menester, por medio de métodos suficientemente operativos e integrados, sustituir las viejas prácticas de gestión en el área de alojamiento turístico. Por ello es necesario desarrollar instrumentos metodológicos que permitan el diseño del plan de negocio como herramienta de gestión para la actividad de tal área, puesto que en la bibliografía consultada y en las investigaciones realizadas los procedimientos que se desarrollan no satisfacen plenamente las exigencias del diseño que se requiere en el contexto del área de alojamiento en este tipo de hoteles.

Diversos autores plantean metodologías con especificidades dependientes de las características de la empresa a la cual están dirigidas (anexo I). A partir del análisis comparativo realizado sobre las diferentes metodologías, procedimientos y pasos estudiados, se pudo determinar que aproximadamente $60 \%$ sitúa el análisis o caracterización de la entidad como una fase inicial en la elaboración del plan de negocio, aunque el resto, de una forma u otra lo incluye dentro de alguna de las primeras etapas. Sin embargo, más de $80 \%$ considera necesaria la elaboración del resumen ejecutivo, a pesar de que algunos lo establecen al inicio y otros al final del documento. Todos los procedimientos contienen el análisis de la estrategia comercial y de la situación económica financiera, por lo que resultan etapas relevantes. Indistintamente, más de $75 \%$ de los autores propone la inclusión de diferentes planes, estrategias, presupuestos y objetivos en sus diferentes etapas, desagregando luego los elementos a tener en cuenta en cada una de ellas. Únicamente $25 \%$ considera importantes los aspectos relacionados con las normativas de funcionamiento, lo cual puede repercutir 
en la calidad del plan una vez concebido y puesto en práctica, mientras que sólo para $30 \%$ es necesaria la inclusión de los recursos humanos. Todas las metodologías analizadas constituyen un aporte desde el punto de vista científico y metodológico, aunque se cree que, por su carácter general, no se adaptan exactamente a la situación planteada en esta investigación; de tal suerte, en este trabajo se plantea una adecuación acorde con las particularidades del objeto de estudio, estableciendo un procedimiento para elaborar el plan de negocio en el área de alojamiento de pequeños hoteles. La especificidad del nuevo procedimiento está dada por la estructura propuesta en cuatro etapas, cada una con sus pasos, lo que permite su mejor consecución, no sólo a la hora de poner en práctica las actividades intrínsecas del plan, sino también en la preparación del personal implicado. Otra peculiaridad es la determinación de la etapa de preparación, la cual no se encontró en ninguna de las metodologías examinadas, sin embargo, se considera de gran relevancia para el éxito del plan, pues de su correcta comprensión y retroalimentación dependerá el resultado final. De hecho, para la elaboración del plan de negocio no es una garantía que la entidad de alojamiento declare su interés por asumir el procedimiento, pues éste puede ser interpretado de diversas formas, lo cual indica la necesidad de fundamentar de manera clara y precisa sus etapas y pasos y su influencia sobre el área de alojamiento del hotel.

El procedimiento consta de cuatro etapas que, al ser integradas correctamente, dan como resultado un acertado plan de negocio para el perfeccionamiento de la gestión de alojamiento del hotel, en virtud del cual se propicia el cumplimiento de los objetivos organizacionales:

Etapa I: Preparación

Etapa II: Orientación estratégica de la gestión de alojamiento

I. Caracterización del área de alojamiento

2. Descripción del servicio de alojamiento

3.Análisis del segmento de mercado

Etapa III: Proyección del plan de negocio

4. Estrategia de comercialización

5. Estructura y gestión de los recursos humanos

6. Información económico-financiera 
Etapa IV: Implementación, control y perfeccionamiento

7. Normativas para el funcionamiento del área de alojamiento

8. Plan de implementación

9. Información adicional

10. Resumen ejecutivo

\section{Etapa I: Preparación}

Tiene como objetivo lograr la familiarización con la entidad, sus procesos y recursos humanos; involucrar al personal del área de alojamiento y capacitarlo para contribuir a la ejecución de las restantes etapas del procedimiento, y programar la ejecución de las etapas subsiguientes. Es una necesidad ineludible que la aplicación del procedimiento parta de conciliar los intereses y compromisos declarados por las partes involucradas (dirección, organizaciones políticas, de masas y especialistas) en un programa de trabajo para el cumplimiento de los objetivos.

\section{Etapa II: Orientación estratégica de la gestión de alojamiento}

Su propósito es asegurar la correspondencia entre las demandas estratégicas del área de alojamiento y las posibilidades de mejora de la calidad de la gestión mediante la implementación del plan. Esta etapa está compuesta por tres pasos: I) se inicia con la caracterización general del área de alojamiento, a partir de la determinación de los elementos que fundamentan su existencia y su desarrollo perspectivo, así como sus objetivos a corto, mediano y largo plazo, los cuales son definidos o actualizados según los resultados del análisis del entorno interno y externo del hotel.2) Se procede a examinar el servicio de alojamiento, haciendo énfasis en el valor distintivo que representa para el cliente, de acuerdo con el segmento de mercado al que va dirigido y las necesidades que satisface. Se pueden destacar los recursos humanos y materiales empleados y el proceso que se utiliza o utilizará. También se incluyen en este paso las proyecciones en la prestación de servicios de alojamiento con que cuenta el hotel. 3) Por último, se analizan los factores que determinan sus niveles de ocupación y las características de sus principales mercados; es decir, cómo influyen en la decisión de compra de los clientes sus preferencias y motivaciones, así como 
el precio, la calidad, las garantías o el servicio de posventa que les ofrece el hotel. Además, se deben considerar las tendencias dominantes en la gestión de alojamiento (cambios en tecnología, productos, mercados, regulaciones o condiciones económicas), y cuáles de ellas podrían incidir en la gestión del área de alojamiento del hotel en los próximos años.

La presencia y uso de estos elementos en el plan de negocio del área de alojamiento permiten desarrollar un pensamiento estratégico y analítico, no en la entidad, sino en el área de alojamiento en particular, para convertirse en el eje central del establecimiento de objetivos y del proceso eficiente de gestión de alojamiento. Los pasos contenidos en esta etapa incluyen a su vez algunos puntos que deben tenerse en cuenta en su desarrollo, y se muestran a continuación.

Paso I. Caracterización del área de alojamiento

- Historia del área de alojamiento del hotel. Describir la firma o el grupo hotelero al cual pertenece, cuándo y por quién fue creado el hotel y los cambios más importantes que hayan ocurrido durante su trayectoria, así como la estructura y las características del funcionamiento del área de alojamiento.

- Objetivos y funcionamiento. Misión y visión a largo plazo de lo que desea el área de alojamiento. En algunos casos, conviene hacer referencia a las estrategias y filosofías de trabajo en el área, para mostrar los esfuerzos que ésta dedica a establecer buenas relaciones con los clientes y con su personal. Es importante fijar cuidadosamente los objetivos que se propone para poder verificar si el área se está desarrollando; en este sentido, deben ser efectivos, simples y mensurables.

- Localización y recursos. Describir dónde se localiza el área de alojamiento y de qué facilidades dispone.

Paso 2. Descripción del servicio de alojamiento

- Descripción de productos o servicios. Describir general y brevemente los productos o servicios que ofrece u ofrecerá el área de alojamiento; cómo serán desarrollados los servicios de reserva, recepción, atención durante la estancia y despedida de los clientes. Pueden destacar los recursos humanos y materiales utilizados y el proceso que se emplea o empleará. 
- Valor distintivo para el cliente. Exponer los aspectos que representan un valor distintivo para el cliente y las necesidades que satisface.

- Perspectivas en la prestación de servicios de alojamiento. Si se tiene planes de actualizar los productos o servicios existentes u ofrecer otros nuevos en los próximos años, explicarlos brevemente. Por ejemplo, aspectos destacables en la capacidad de gestión del producto habitación, que puede significar una ventaja con respecto a sus competidores, como personal especializado, nueva tecnología, insumos a menores costos, etcétera.

\section{Paso 3. Descripción del segmento de mercado}

- Estudios de mercado. Comentar si en el hotel se ha efectuado alguna investigación del mercado en que se desenvuelve. Describir características y factores que determinan esa investigación, niveles de ocupación y mercados principales, preferencias y motivaciones. Explicar los servicios que integran el área de alojamiento, destacando el tamaño y las particularidades de los segmentos en los que el hotel deberá competir. Identificar el mercado en grupos de clientes, enfatizando sus características y tamaño, según la estacionalidad.

- Proceso y criterios de compra de los clientes. Saber por qué los clientes deciden alojarse en el hotel y no en los de la competencia: por cuestiones de precio, calidad, garantías, servicio de posventa, etcétera.

- Tendencias clave en el sector hotelero respecto al alojamiento. Explicar brevemente las tendencias dominantes en la gestión de alojamiento (cambios en tecnología, productos, moda, mercados, regulaciones o condiciones económicas) y cuáles de ellas podrían influir en los próximos años. Considerar qué mercados tienen las mayores oportunidades de crecimiento en el futuro y por qué; para cuáles se puede esperar una declinación en las ventas y qué acciones potenciar al respecto. Los elementos que forman parte de esta etapa del procedimiento fomentan el desarrollo de acciones que contribuyen a que:

a) Todos los miembros de la entidad conozcan los elementos que orientan y conducen la gestión de alojamiento

b) Cada individuo sepa en qué forma sus esfuerzos individuales se combinan e integran al esfuerzo de otros, a fin de contribuir colectivamente al logro de objetivos comunes 


\section{Etapa III: Proyección del plan de negocio}

Para desarrollar un cambio satisfactorio que permita planear con eficiencia los procesos de alojamiento según el procedimiento propuesto, es necesario estudiar y definir las posibles adecuaciones que, en el contenido de las funciones y procesos internos, debe asumir el área de alojamiento para garantizar una correspondencia entre los objetivos fijados y los mecanismos determinados para su logro. El objetivo fundamental de esta etapa es describir las estrategias de comercialización que establece el hotel para su producto alojamiento, y en función de ello determinar cualitativa y cuantitativamente las necesidades financieras y de personal, así como la programación de las acciones requeridas para asegurar la obtención, mantenimiento y mejora del personal con las competencias necesarias para la gestión de alojamiento, de manera que se garantice el desarrollo eficaz del plan y el logro de los beneficios esperados. Esta etapa se llevará a cabo mediante la consecución de tres pasos establecidos a partir de la etapa anterior, los cuales incluyen una serie de elementos que se describe a continuación.

Esta tercera etapa parte del análisis de la posición competitiva del área de alojamiento, previendo cómo los mercados pueden cambiar durante el periodo del plan de negocio. Se realiza un estudio de la estructura y organización de los recursos humanos, dada la necesidad de garantizar el personal disponible habitualmente y su proyección en los próximos años. Se describen las principales funciones y responsabilidades de cada uno de los puestos y personas en el área de alojamiento. Por último, se determina la cantidad de dinero necesaria y las utilidades esperadas por concepto de alojamiento.

Paso 4. Estrategia de comercialización

- Mercado objetivo. Definir el mercado objetivo por tipo de cliente y por región. Prever cómo los mercados pueden cambiar durante el periodo del plan de negocio. Diseñar los posibles escenarios.

- Descripción de los principales competidores. Enumerar los principales competidores y proporcionar una descripción abreviada de los servicios en términos de la localización, producción, estrategias de comercialización y posición en el mercado.

- Análisis de la posición competitiva. Identificar las ventajas competitivas del área de alojamiento sobre sus competidores, así como las desventajas. 
- Estrategia de precios. Cómo están establecidos los precios de productos o servicios y cómo son en relación con los de los competidores.

- Estrategia de comunicación y promoción. De qué manera se dan a conocer los productos o servicios a los mercados. Dónde están ubicados los clientes y cómo se llegará a ellos, tanto para la venta como para la posventa. Destacar las actividades que se emprenderán con ese fin.

\section{Paso 5. Estructura y gestión de los recursos humanos}

- Estructura. Describir la organización del área de alojamiento; de cuánto personal dispone habitualmente y cuánto debe tener en los próximos años; las principales funciones y responsabilidades de cada uno de los puestos y personas.

- Personal de gerencia. Quién o quiénes integran el equipo de dirección. Listar con una breve descripción del cargo que ocupa cada persona, sus funciones principales y la experiencia en cada caso.

- Personal. Explicar si se necesita personal; cómo se cubrirán los cargos que no son de dirección en el área de alojamiento, señalando el perfil y nivel de experiencia requeridos, los salarios estimados $y$, en caso de que este personal precise algún tipo de entrenamiento, si se está en condiciones de suministrarlo o se acudirá a la capacitación externa.

- Mercado de trabajo. Reconocer qué factores pueden afectar la capacidad de identificar el personal que se necesita y mantenerlo en el área de alojamiento.

- Métodos de trabajo. Explicar si el hotel puede variar el método de trabajo o prestación del servicio de alojamiento y si ha hecho estimaciones del costo de esa variación.

Paso 6. Información económico-financiera

- El área de alojamiento puede desarrollarse y sobrevivir sólo si genera utilidades para el hotel. En la etapa de proyección del plan de negocio se debe saber la cantidad de dinero necesaria y las utilidades esperadas. Para ello, la información financiera básica que debe contener el plan de negocio comprende cuatro documentos principales: 
a) Flujos financieros mensuales para el primer año fiscal, que mostrarán cuántos fondos ingresarán y cuántos egresarán

b) Flujos financieros anuales para el segundo y tercer años fiscales

c) Balance proyectado a tres años

d) Estado de resultados proyectado a tres años

- En el balance se requiere una información como punto de partida. Debe elaborarse una planilla que contenga los datos del balance inicial para el periodo correspondiente al plan de negocio considerado. Además de las cifras contenidas en el balance, interesa conocer lo siguiente:

a) Situación a la fecha de la presentación del plan

b) Plazos de cobros y pagos

c) Principales acreedores

d) Principales contingencias incorporadas al balance

e) Factores que han alterado su rentabilidad

El balance general proyectado es básico para conocer la situación financiera al término de cada año, en especial la relación activo-pasivo del hotel. El estado de resultados anual reúne la información que ha sido preparada previamente, a la que pueden agregarse los intereses de cada operación de crédito que se haya efectuado, las amortizaciones y los impuestos correspondientes a cada año proyectado. Con la integración de los elementos propuestos en esta etapa del procedimiento se garantiza un elevado nivel de correspondencia entre los recursos más importantes a la hora de poner en práctica el plan de negocio, es decir, recursos humanos, financieros, $y$ el cliente como eje fundamental en la gestión de alojamiento del hotel.

\section{Etapa IV: Implementación, control y perfeccionamiento}

Esta etapa tiene como objetivos principales mantener el control sobre la implementación de lo proyectado en la etapa anterior, determinar posibles desviaciones y evaluar los ajustes necesarios. En esta última parte, el procedimiento relaciona las vías y formas de control requeridas para cada etapa y paso 
establecidos, permitiendo con su integración, al cierre de un periodo de trabajo, evaluar los resultados obtenidos, según el nivel de cumplimiento de los objetivos definidos con la aplicación del procedimiento, y retroalimentando la puesta en marcha del plan de negocio en el área de alojamiento, proceso que le confiriere al procedimiento una mayor flexibilidad y adaptabilidad en su implementación.

En esta fase, la gerencia del hotel debe brindar el máximo apoyo al plan; debe entender que el plan de negocio es una herramienta para la gestión sobre bases diarias, más que un ejercicio académico de escribir objetivos una vez al año. Por ello, cada etapa establece los pasos necesarios para el cumplimiento de los objetivos del área de alojamiento; elementos con los cuales, en cada periodo, se comparan los resultados alcanzados y se determinan las formas de estimulación y reconocimiento por el desempeño realizado.

El procedimiento plantea un control, cuya finalidad es lograr la superación y el perfeccionamiento de la gestión de alojamiento con el uso del plan de negocio. Una parte importante en esta etapa final es elaborar el resumen ejecutivo, el cual debe incluir los aspectos más relevantes del plan, e irá al inicio del documento. Los pasos en esta etapa y sus respectivos elementos se exponen a continuación.

Paso 7. Normativas para el funcionamiento del área de alojamiento

- Documentos normativos. Qué tipo de disposiciones normativas pueden afectar la actividad de alojamiento en forma directa. Licencias o permisos requeridos para su funcionamiento. Qué medidas se han considerado para el adecuado cumplimiento de las normativas.

- Protección. Sistemas de protección o mecanismos empleados con este fin en el área de alojamiento.

Paso 8. Plan de implementación

- Inicio de las actividades del plan de negocio. Señalar cuándo se iniciarán las actividades contenidas en el plan de negocio y quiénes serán los responsable de llevarlas a cabo, así como cuándo finalizarán. El plan debe ser flexible, para permitir la retroalimentación.

- Riesgos. Identificar si existe alguna situación que pueda afectar la demanda de los productos o servicios de alojamiento durante la continuación del plan de negocio. Diferenciar dos tipos de riesgos: 
los propios del sector y los intrínsecos del área de alojamiento y el hotel en sí. En caso de considerar algún otro riesgo (que no esté relacionado con el sector) que pueda afectar el éxito de la gestión de alojamiento, indicar cómo se prevé atenuar su impacto.

- Estrategias de contingencia. En el plan de negocio se deben incluir posibles estrategias de contingencia en caso de que el área de alojamiento no alcance los objetivos previstos.

Paso 9. Información adicional

- Si se cree conveniente, se puede agregar a continuación o adjuntar como anexo la información que se considere de importancia respecto al hotel, el área de alojamiento y el plan de negocio, o aquella adicional de carácter técnico, promocional, económico-financiero, entre otra.

Paso 10. Resumen ejecutivo

- Esta parte del plan de negocio sólo puede ser preparada cuando finalice la elaboración de éste, aunque debe ser colocada al principio del documento, es decir, antes de la descripción del plan de negocio e inmediatamente después de la carátula, $y$ debe contener lo siguiente:

a) Breve caracterización del área de alojamiento

b) Descripción del servicio de alojamiento

c) Principales oportunidades de mercado

d) Elementos fundamentales de la estrategia de comercialización

e) Estructura y gestión de los recursos humanos

f) Información económico-financiera

g) Principales documentos normativos del área de alojamiento

- Las explicaciones que se brindan en las diferentes etapas del plan no sólo deben reflejar que se conoce bien la gestión de alojamiento, sino también su relevancia para el hotel, lo cual únicamente pueden hacerlo las personas encargadas de prestar el servicio. La elaboración del plan de negocio aplicando el procedimiento propuesto constituye una alternativa para el perfeccionamiento de la gestión de alojamiento en pequeños hoteles, pues contribuye, entre otros aspectos, a lograr: 
a) Una finalidad más precisa

b) El incremento de la motivación por el trabajo de directivos y mandos intermedios

c) La autodirección por parte de los trabajadores

d) Un ambiente orientado al cumplimiento

e) Una mejora de la comunicación y la cooperación

\section{Conclusiones}

I. El procedimiento propuesto para elaborar el plan de negocio del área de alojamiento en pequeños hoteles se realiza sobre la base del análisis comparativo de las metodologías planteadas por los autores estudiados, adecuándolas a las condiciones examinadas del objeto de estudio.

2. El procedimiento propuesto considera para su ejecución las últimas tendencias en la elaboración de un plan de negocio, referidas por los autores estudiados a efectos de esta investigación, asegurándose la derivación de las competencias necesarias, $y$, de modo implícito, se crean las bases para que el área de alojamiento desarrolle cabalmente los principios y procesos que caracterizan a la organización que aprende, a partir de la determinación de las necesidades actuales de los establecimientos de alojamiento turístico y las previsiones, elementos que se han tenido en cuenta en el diseño de las etapas y los pasos del procedimiento descrito.

3. La estructuración del procedimiento planteado en cuatro etapas y diez pasos para su diseño e implementación permite realizar un proceso complejo de forma relativamente sencilla, lo que resalta su carácter práctico, minimizando las complejidades y deficiencias detectadas en las metodologías estudiadas.

4. El plan de negocio requiere una indispensable preparación en los conceptos, principios y etapas que establece el procedimiento, de tal forma que comience a desarrollarse una filosofía de gestión participativa orientada a la eficiencia del área de alojamiento y la satisfacción de los clientes. 


\section{FUENTES CONSULTADAS}

Álvarez, C. J. L. (200I). “El nacimiento del turismo moderno”. Revista Conocer, 2, 6-12. México.

Cruz, R. S. (2007). Desarrollo de un plan de negocio. ¿Cómo iniciar su empresa? [en línea]. Disponible en: http://www.monografias.com/trabajos35/ plan-de-negocio/plan-de-negocio.shtml?monosearch

Dezerega, C.V. (2004). Plan de negocios (Business Plan): un imperativo gerencial [en línea]. Disponible en: http://www.gestiopolis.com/recursos/ documentos/fulldocs/emp/plandez.htm

Díaz, D. S. (1994). El plan de negocios. Un sistema de trabajo imprescindible para facilitar la continuidad, desarrollo, crecimiento y rentabilidad de su empresa. Guías de gestión de la pequeña empresa. Madrid: Díaz de Santos.

Figuerola, P. M. (1990). Elementos para el estudio de la economía de la empresa turística. Selección de contenidos y síntesis del libro. Maestría en Gestión Turística. La Habana: Centro de Estudios Turísticos-Universidad de La Habana.

Fontanez, D. (2005). Cómo elaborar un plan de negocios [en línea]. Disponible en: http://www.gestiopolis.com/canales5/comerciohispano/ I I8.htm Gallegos, J. F. (2002). Gestión de hoteles, una nueva visión. Madrid: Paraninfo.

González Valdés, B. L. (2008). Diagnóstico actual y perspectivo de la situación del alojamiento turístico en Villa Clara. Informe de investigación terminada. Santa Clara: Centro de Estudios Turísticos (Cetur)-Universidad Central "Marta Abreu" de Las Villas (UCLV).

González Valdés, B. L. y C. C. Martínez Martínez (2008). Experiencias en el diseño y aplicación de un procedimiento para elaborar el plan de negocio del área de alojamiento en pequeños hoteles de tránsito. Santa Clara: Tercer Taller Internacional de Hotelería y Turismo (Hoteltur 2008). (2009). Procedimiento gerencial para la elaboración del plan de negocio del área de alojamiento de pequeños y medianos hoteles de tránsito categoría tres estrellas. Informe de investigación terminada. Santa Clara: Cetur-uClv.

González Valdés, B. L. y M. Y. Moya (2007). “Diseño y aplicación de un procedimiento para la elaboración del plan estratégico en entidades turísticas".Tercer Encuentro de la Red Iberoamericana de Evaluación 
y Decisión Multicriterio (RED-M). Culiacán:Universidad de Occidente/ Universidad Autónoma de Sinaloa, 5-8 de noviembre de 2007.

González Valdés, B. L., M.Y. Moya y C. C. Martínez Martínez (2008). “Procedimiento para el plan estratégico en entidades turísticas de Villa Clara”. Retos Turísticos, 7 (3), 35-39. Universidad de Matanzas.

Hernández, M. R. y L. del C. Zaragoza (2004). Plan de negocios para la creación del aserradero "San Felipe Maderas". Tesis de licenciatura en Administración de Empresas. Puebla: Departamento de Administración de Empresas-Escuela de Negocios-Universidad de las Américas.

Herrera, L. K. (2000). Sistema de Gestión Participativa por Objetivos con Enfoque Estratégico: aplicación en pequeñas y medianas instalaciones turísticas hoteleras. Resumen de tesis para obtener el grado de doctora en Ciencias Técnicas. Santa Clara: UCLV.

Infante, N. M. y T. R. Carballosa (2004). Principales consideraciones sobre el plan de negocios [en línea]. Disponible en: http://www.monografias.com/ trabajos I5/plan-negocio/plan-negocio.shtml?monosearch

Lattin, G. W. (2000). "Desarrollo de la industria hotelera”, en G. W. Lattin. Administración moderna de hoteles y moteles. México:Trillas, I3-64.

Lynn,J.(2000). Plan de negocios [en línea]. Entrevista aVicky Helmick, especialista y consultora de empresas. Ingeniería comercial. Disponible en: http:// www.ingenieriacomercial.com/portal/Emprendedores/Plan-deNegocios---Business-Plan--Como-empezar.html

Martín, F. R. (2003). Manual de teoría y práctica del turismo. La Habana: Centro de Estudios Turísticos-Universidad de La Habana.

Martínez Martínez, C. C. (2003). El enfoque en sistema:su aplicación en el análisis de las micro, pequeñas y medianas empresas (Pymes) turísticas. Ocotlán: Centro Universitario de La Ciénega-Universidad de Guadalajara. (2007). Manual de preparación de pequeños proyectos empresariales. Puyo: Universidad Estatal Amazónica.

Martínez Martínez, C. C., B. L. González Valdés y M. Y. Moya (2006). "Procedimiento para el diagnóstico estratégico en la micro, pequeña y mediana empresa turística: Experiencias de aplicación”, en Memorias de la V Conferencia Internacional de Ciencias Empresariales. II Taller Internacional de Hotelería y Turismo (Hoteltur 2006). Octubre de 2006. Santa Clara: Feijóo. 
Martínez Martínez, C. C., P. O.Valdivia y S. Y. Cruz (2007). El plan de negocios; una técnica para la gestión de empresas turísticas [en línea]. Disponible en: http://www.monografias.com/trabajos43/plan-de-negocios/plande-negocios.shtml

Matos, R.H.(2005). Turismo. Complete su conocimiento. Intermediación/Distribución turística. Pinar del Río: Escuela de Hotelería y Turismo Varadero.

Muñoz, E. F. (2004). La industria turística [en línea]. Disponible en: http://www. eumed.net/cursecon/libreria/

Nueno, P. (1992). Emprendiendo. El arte de crear empresas y sus artistas. Madrid: Deusto.

Pereira, J. E. (2006). El plan de negocios [en línea]. Disponible en: http://www. gestiopolis.com/canales $6 / \mathrm{mkt} / \mathrm{mercadeopuntocom} / \mathrm{el}$-plan-denegocio.htm

Terragno, D. y M. L. Lecuona (2006). Cómo armar un plan de negocios. Caracas: Dinero.

Torres Márquez, A. C. (2007). "El plan de negocios, una herramienta poco utilizada”. Betsime, julio-septiembre de 2007 [en línea]. México: Instituto Politécnico Nacional. Disponible en: http://www.betsime. disaic.cu/secciones/mer_julsep_07.htm

Wald, A. (2005). ¿Quién necesita un plan de negocio? [en línea]. Disponible en: http://www.gestiopolis.com/Canales4/Wald/8I.htm 
Anexol. Cuadro comparativo de metodologías propuestas por los diferentes autores sobre plan de negocio

\begin{tabular}{|c|c|c|c|c|c|c|}
\hline $\begin{array}{l}\text { Autores } \\
\text { Elementos }\end{array}$ & $\begin{array}{l}\text { Nueno, } \\
1992\end{array}$ & $\begin{array}{l}\text { Infante, } \\
2004\end{array}$ & $\begin{array}{l}\text { Dezerega, } \\
2004\end{array}$ & $\begin{array}{l}\text { Fontanez, } \\
2005\end{array}$ & $\begin{array}{l}\text { Pereira, } \\
2006\end{array}$ & $\begin{array}{l}\text { Martínez, } \\
2007\end{array}$ \\
\hline Caracterización de la entidad & & $\mathrm{X}$ & $\mathrm{X}$ & $\mathrm{X}$ & $x$ & \\
\hline $\begin{array}{l}\text { Descripción del producto y / o } \\
\text { servicio }\end{array}$ & & $x$ & $x$ & $x$ & $x$ & $x$ \\
\hline Análisis del segmento de mercado & $x$ & & & $x$ & $x$ & $x$ \\
\hline Estrategia de comercialización & $x$ & $x$ & $x$ & $x$ & $x$ & $x$ \\
\hline Análisis económico-financiero & $x$ & $x$ & $x$ & $x$ & $x$ & $x$ \\
\hline Normativas para funcionamiento & $x$ & & & & $x$ & $x$ \\
\hline Plan de implementación & $x$ & & $x$ & & & $x$ \\
\hline Información adicional & & $x$ & & $x$ & & \\
\hline Resumen Ejecutivo & $\mathrm{X}$ & $\mathrm{X}$ & & $\mathrm{X}$ & & $x$ \\
\hline
\end{tabular}

Fuente: Elaborado por los autores a partir de la bibliografía consultada 
